\title{
Research On The Estimation Of Lithium Battery SOC Based On EKF
}

\author{
Zhi-sheng $\mathrm{An}^{\mathrm{a}}$, Zhi-yi Sun ${ }^{\mathrm{b}}$ and Quu-sheng He \\ Taiyuan university of science and technology, Taiyuan city 030024, China

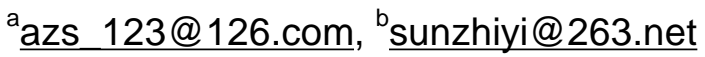

Keywords: Lithium-ion battery; State of charge; Extended Kalman filter; Battery model.

\begin{abstract}
In the battery management system, the SOC estimation is the most basic and most important part. The exact estimation of SOC can prevent battery over-change or over-change, and extend battery life. Based on traditional SOC estimate algorithm and analysis of factors affecting SOC, the method to estimate stalling state and charge-discharge state of battery respectively is applied to predict SOC of lithium-ion battery. Especially in charge-discharge state, extended Kalman filter is applied in estimation. A state space model of a lithium-ion battery based on Thevenin model is established, which has the advantage of simplicity and could be easily implemented. Matlab simulation and experiments were carried out. Comparison indicates that performance of the model accords well with that of lithium-ion battery. The extended Kalman filter keeps an excellent precision in full range of the SOC, and performs well when initial error of disturbance happens.
\end{abstract}

\section{Introduction}

With the progress of science and technology, lithium-ion battery has been used in industry, daily life and other fields widely. So it is important to require the SOC accurately and reliably. Because of the particularity of lithium-ion battery, estimating the SOC accurately has great difficulties.

Estimation of SOC has many methods, such as accumulated power method, open-circuit voltage, the method of resistance, Kalman filter, neural network and fuzzy reasoning and so on. In the present application, open-circuit voltage method is appropriate for a management system which demands low estimated precision and accumulated power method for higher estimated precision. During the application of accumulated power method, the later correction is important, which usually uses the method of combining voltage calibration and open-circuit voltage method.

A method, which divided the working conditions into stalling and charge-discharge state, is used to estimate SOC according to the practical situation of lithium battery in this paper.

\section{Influencing factors of the SOC estimation}

The battery state of charge is difficult to be estimated, as it is influenced by many kinds of factors, such as charging and discharging current, battery internal temperature, self-discharge, aging and so on. Most of the factors influence estimation of SOC through the polarization effect. The polarization effect can influence voltage and electric quantity of the battery. So the influence caused by polarization effect must be considered. Otherwise, it will produce accumulative error and incorrect SOC value.

Coulomb efficiency, $\eta$, is the parameter, which could represent the influence aroused by polarization effect and the charging and discharging efficiency. Its value is influenced mainly by the current and the battery temperature.

\section{Estimation of the SOC}

Working conditions of the battery could be divided into stalling and charge-discharge state according to the actual situations. Corresponding method is made to estimate SOC based on different state.

Stalling state. The stalling state is defined as the state of battery which does not include charge-discharge state. The SOC value at this moment can be used as the SOC initialization value of 
the charge-discharge state. Experiments prove that SOC value and open-circuit voltage have corresponding relationship. So open-circuit voltage is used to estimate SOC by their relationship.

SOC is influenced by the length of time, which is in stalling state. The battery voltage has not recovered fully, if the battery rests for a short time. Recovery of the electric quantity increases as the time goes and is different depended on the battery type and degree of polarization. If lithium-ion battery is rested for two hours, its polarization effect can disappear.

So if the time that battery rests for is longer than two hours, SOC value can be obtained based on the open-circuit voltage at this moment. Otherwise, it is referred to Eq. 1.

$$
\operatorname{SOC}(t)=K_{r} \operatorname{SOC}_{\text {open }}(t)+\left(1-K_{r}\right) \operatorname{SOC}\left(t_{0}\right)
$$

In Eq. 1, $\operatorname{SOC}_{\text {open }}(t)$ is initial electric quantity that is based on the OCV in the time of $\mathrm{t}$ and $\operatorname{SOC}\left(t_{0}\right)$ is the electric quantity in the time of $t_{0}$ when changing and discharging has stopped. Constant $K_{r}$ characters the relationship between initial SOC and battery recovery effect.

Charge-discharge state. Estimation during the charge-discharge state is important and difficult. The battery voltage, current, and the temperature of the state change violently. Therefore, the extended Kalman filter algorithm, which is combined with accumulated power method, is used to obtain a more accurate SOC value.

Battery model. Kalman filtering algorithm is a optimization algorithm which makes optimum estimation in the sense of the minimum variance for a state of the power system. Battery model suitable for Kalman filtering algorithm must have the following features. (1)It can reflect the dynamic performance of the battery well. (2)Order of the model should not be too high, for it can reduce the processor operation.

Thevenin model can represent static and dynamic characteristics of the battery. Fig. 1 is the Thevenin model of battery.

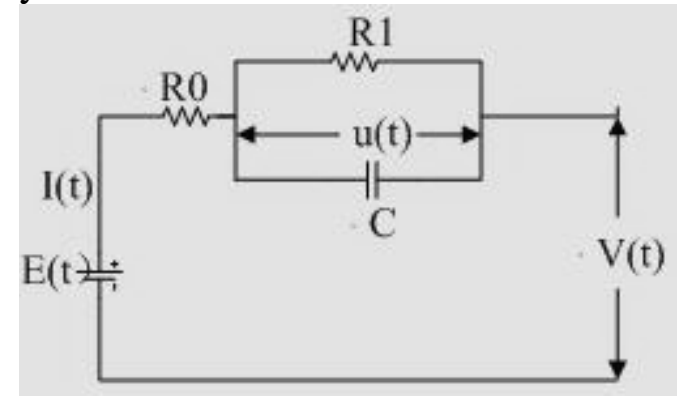

Fig. 1 Thevenin model

EKF estimate strategy based on the model of Thevenin. The following mathematical equations could be established from the Thevenin model.

$$
\begin{aligned}
& E(t)=V(t)+R_{0} I(t)+u(t) \\
& I(t)=\frac{u(t)}{R_{1}}+C \frac{d u}{d t} \\
& E(t)=F(\operatorname{SOC}(t))
\end{aligned}
$$


$\operatorname{SOC}(t)=\operatorname{SOC}\left(t_{0}\right)-\frac{1}{C_{N}} \int_{t_{0}}^{t} \eta I d t$

In Eq. 5, $C_{N}$ is the battery rated power. State and measurement equations are reasoned from Eq. 2, Eq. 3, Eq. 4 and Eq. 5.

$$
\begin{aligned}
& {\left[\begin{array}{l}
S O C_{k+1} \\
u_{k+1}
\end{array}\right]=\left[\begin{array}{lc}
1 & 0 \\
0 & \exp \left(\frac{-\Delta t}{R_{1} C}\right)
\end{array}\right]\left[\begin{array}{l}
S O C_{k} \\
u_{k}
\end{array}\right]+\left[\begin{array}{l}
-\frac{\Delta t}{C_{N}} \eta \\
R_{1}\left(1-\exp \left(\frac{-\Delta t}{R_{1} C}\right)\right)
\end{array}\right] \cdot \boldsymbol{i}_{k}+\left[\begin{array}{l}
w_{1}(k) \\
w_{2}(k)
\end{array}\right]} \\
& V_{k}=F\left(S O C_{k}\right)-R_{0} i_{k}-\boldsymbol{u}_{k}+v(k)
\end{aligned}
$$

In Eq. $6, \mathcal{W}_{1}^{(k)}$ and $\mathcal{W}_{2}^{(k)}$ are the interference that some unmeasured random inputs make. $v(k)$ in Eq. 7 is the measurement noise from the battery voltage. State variable, $\mathrm{X}(\mathrm{k})$, includes $S O C_{k}$ and $\boldsymbol{U}_{k}$. Matrix A, B, C(k) can be obtained after the linearization of Eq. 7.

$$
\begin{gathered}
A=\left[\begin{array}{cc}
1 & 0 \\
0 & \exp \left(\frac{-\Delta t}{R_{1} C}\right)
\end{array}\right] . \\
B=\left[\begin{array}{l}
-\frac{\Delta t}{C_{N}} \eta \\
R_{1}\left(1-\exp \left(\frac{-\Delta t}{R_{1} C}\right)\right)
\end{array}\right] \\
\mathrm{C}(\mathrm{k})=\left[\begin{array}{ll}
\frac{\partial F\left(S O C_{k}\right)}{\partial S O C_{k}} & -1
\end{array}\right] X(k)=\hat{X}(k \mid k-1)
\end{gathered}
$$

In Eq. 10, $\hat{X}(k \mid k-1)$ is the predictive value of state variable. The following equations are the optimal formula of Kalman filter for discrete system.

$$
\begin{aligned}
& \hat{X}(k \mid k-1)=A \hat{X}(k-1)+B I(k-1) \\
& P(k \mid k-1)=A P(k-1) A^{T}+Q \\
& K(k)=P(k \mid k-1) C^{T}(k)\left[C(k) P(k \mid k-1) C^{T}(k)+r\right]^{-1}
\end{aligned}
$$




$$
\begin{aligned}
& \hat{X}(k)=\hat{X}(k \mid k-1)+K(k)[V(k)-\hat{V}(k)] \\
& P(k)=[I-K(k) C(k)] P(k \mid k-1)
\end{aligned}
$$

In above formulas, $\mathrm{P}(\mathrm{k}-1)$ and $\mathrm{P}(\mathrm{k} \mid \mathrm{k}-1)$ are filtering error and variance array of prediction error respectively, $\mathrm{K}(\mathrm{k})$ for filtering gain. $\mathrm{Q}$ and $\mathrm{r}$ are variance array of noise $\mathrm{w}(\mathrm{k})$ and variance of $\mathrm{v}(\mathrm{k})$ respectively. Estimated value is obtained by the above optimal formulas of Kalman filter combined with matrix $\mathrm{A}, \mathrm{B}, \mathrm{C}(\mathrm{k})$.

\section{SOC simulation based on Kalman filtering estimate}

Model simulation. In order to verify the performance of the battery model, the experiment on electric discharge of the battery was conducted in room temperature. The parameters of the model would be set as constants in the laboratory.

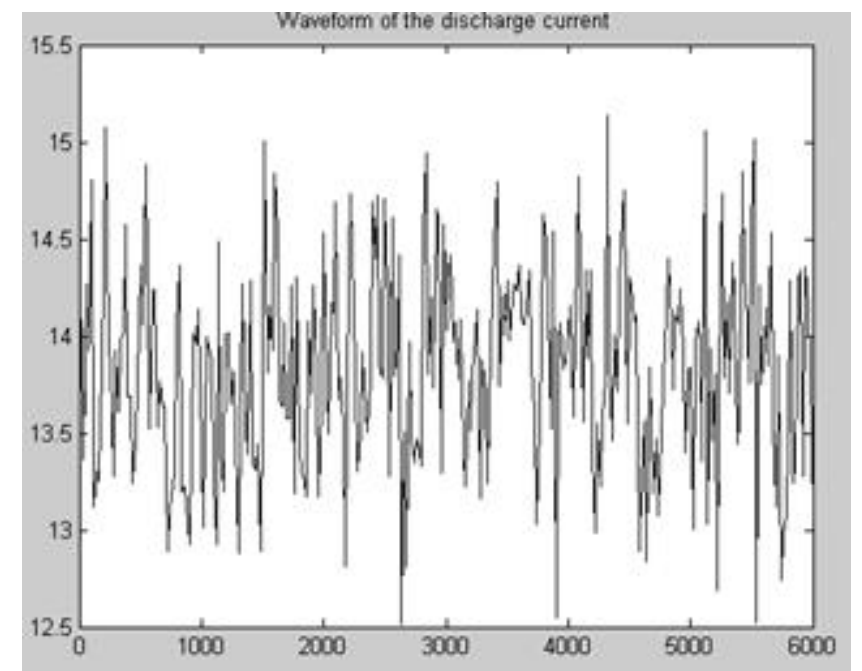

Fig. 2 Waveform of the discharge current

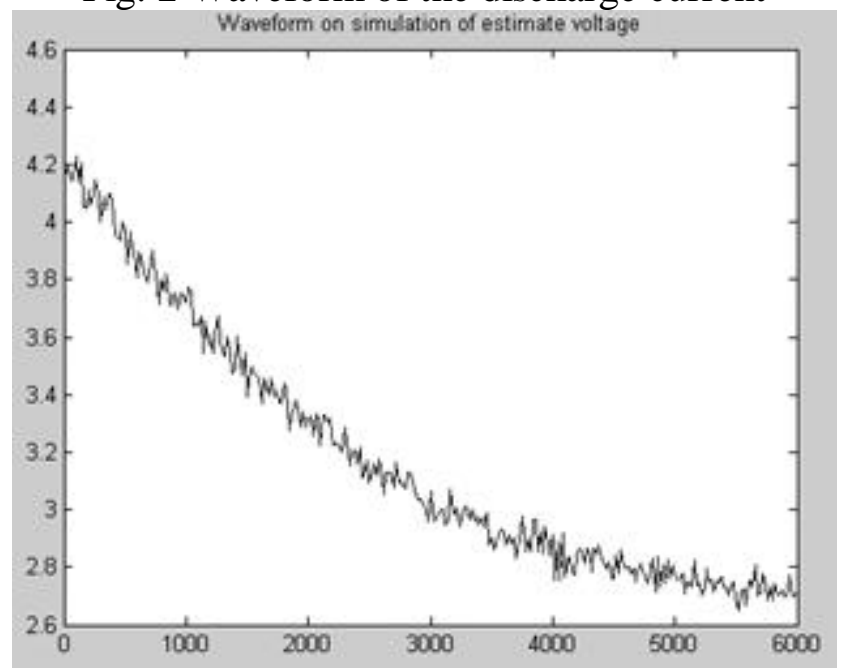

Fig. 3 Waveform on simulation of estimate voltage

Fig. 2 is the waveform of the discharge current during the experiment. Fig. 3 shows variation of the simulation voltage based on the excitation of the above discharge current. The measured voltage of the battery was in good conformity with the voltage of the simulation model. So the model of Thevenin can show the true nature of the battery well. 
Simulation of SOC based on EKF. The dotted line in Fig. 4 is the curve of SOC analog output, and the solid line for the curve of SOC estimation based on extended Kalman filter. The simulation results show that Kalman filter has the very strong convergence. Error caused by initial SOC value and Gaussian white noise can be eliminated by Kalman filter.

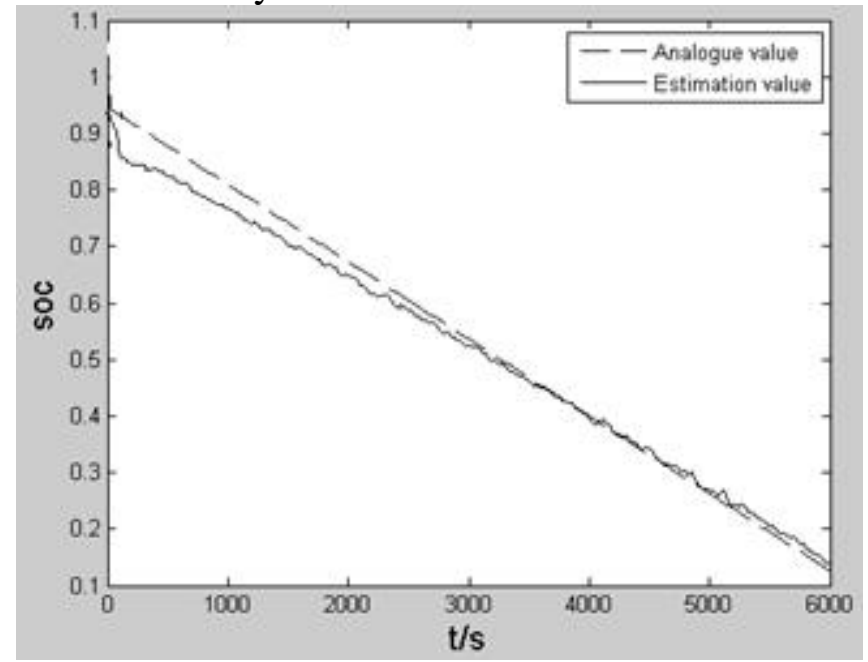

Fig. 4 Simulation diagram of SOC

\section{Conclusion}

In this paper SOC was estimated through two different states of battery. Particularly in charge-discharge state, extend Kalman filtering method based on traditional accumulated power method was used to monitor the changes of SOC in real time. It was prove to be feasible based on the simulation results. But the precision of the algorithm depends on the accuracy of battery model. Establishment of accurate model and measurement of the parameter values are important. However, the parameter value varies as life of battery changes. It is connected with current, temperature and SOC value. So online identification of the parameters needs to be researched further.

\section{References}

[1] Xiong Rui, Sun Fengchun and He Hongwen: Chinese High Technology Letters, Vol. 22 (2012) No. 2, p. 198.

[2] Sun Jun, Li Baohui and Xue Min: Auto Engineer, China, (2011) No. 12, p. 25.

[3] Zhang Di, Ma Yan and Bai Qingwen: Automobile Technology, China, (2011) No. 8, p. 42.

[4] Fan Bo, Tian Xiaohui and Ma Jianwei: Chinese Journal Of Power Sources, Vol. 34 (2010) No. 8, p. 797.

[5] Liu Baojie, Wang Yan and Yin Tianming: Electrical automobile, China, Vol. 32 (2010) No. 1, p. 60 .

[6] Li Bingyu, Chen Xiaodong: Chinese Journal Of Power Sources, Vol. 34 (2010) No. 9, p. 931.

[7] Sun Jingxia, Tan Derong: Agricultural equipment and vehicle engineering, China, (2010) No. 9, p. 20.

[8] Xia Chaoying, Zhang Shu and Sun Hongtao: Chinese Journal Of Power Sources, Vol. 31 (2007) No. 5, p. 414.

[9] Wang Junping, Cao Binggang and Chen Quanshi: Chinese Journal Of Mechanical Engineering, Vol. 44 (2008) No. 5, p. 76. 